\title{
Reconsolidation of episodic memories: A subtle reminder triggers integration of new information
}

\author{
Almut Hupbach, ${ }^{1,3}$ Rebecca Gomez, ${ }^{1}$ Oliver Hardt, ${ }^{2}$ and Lynn Nadel ${ }^{1}$ \\ ${ }^{1}$ Department of Psychology, The University of Arizona, Tucson, Arizona 85721, USA; ${ }^{2}$ Department of Psychology, McGill \\ University, Montreal, Quebec H3A 2T5, Canada
}

\begin{abstract}
Recent demonstrations of "reconsolidation" suggest that memories can be modified when they are reactivated. Reconsolidation has been observed in human procedural memory and in implicit memory in infants. This study asks whether episodic memory undergoes reconsolidation. College students learned a list of objects on Day 1. On Day 2, they received a reminder or not, and then learned a second list. Memory for List 1 was tested immediately on Day 2 (Experiment 2) or on Day 3 (Experiment 1). Although the reminder did not moderate the number of items recalled from List 1 on either day, subjects who received a reminder incorrectly intermixed items from the second list when recalling List 1 on Day 3. Experiment 2 showed that this effect does not occur immediately and thus is time-dependent. The reminder did not affect memory for List 2 on Day 3 (Experiment 3), demonstrating that modification occurred only for the original memory (List 1). The study demonstrates the crucial role of reminders for the modification of episodic memory, that reconsolidation of episodic memory is time-dependent, and, in contrast to previous reconsolidation findings, that reconsolidation is also a constructive process, one that supports the incorporation of new information in memory.
\end{abstract}

Consolidation theory assumes that memories are labile during a limited window after encoding, but as time passes, memories are consolidated and become resistant to change (e.g., McGaugh 2000). The rediscovery of reactivation-induced reconsolidation has challenged this view (cf. Przybyslawski and Sara 1997; Sara 2000; Nader 2003; cf. Lewis et al. 1968; Misanin et al. 1968, for early demonstrations of the reconsolidation phenomenon). In contrast to the consolidation account, reactivation is thought to return memories to a labile state, which allows them to change. In the normal course of events, reactivated memories are retained through a process similar to initial consolidation, i.e., reconsolidation. This view of memory formation as a dynamic process rather than a static one has implications for theories of memory and for our understanding of memory change.

Nader et al. (2000) showed that memories are labile by demonstrating that fear memory in the rat can be severely impaired at the time the memory is recalled. Rats received several tonefootshock pairings on Day 1. The following day, half of the animals were again exposed to the tone. The tone elicited freezing, indicating that the rats recalled the conditioned fear memory. All rats were then injected with a protein-synthesis inhibitor or its vehicle into the amygdala. When tested for fear memory in response to the tone on Day 3, rats who had received the reminding tone before injection of the inhibitor showed less freezing than the vehicle-injected rats and, most importantly, than rats who were not reminded. This demonstrated that reactivation of the fear memory returned the memory to a labile state, which like a newly acquired, nonconsolidated memory requires de novo protein-synthesis in order to be maintained. The proteinsynthesis inhibitor blocked reconsolidation, and thus led to memory impairment. This finding has now been replicated in a variety of species and paradigms (see Dudai and Eisenberg 2004; Alberini 2005).

Walker et al. (2003) recently demonstrated reconsolidation

\footnotetext{
${ }^{3}$ Corresponding author.

E-mail ahupbach@email.arizona.edu; fax (520) 621-9306.

Article published online in January 2007. Article and publication date are at http://www.learnmem.org/cgi/doi/10.1101/lm.365707.
}

effects in humans. Participants were trained on a procedural motor-skill task that involved finger-tapping a simple sequence (e.g., 4-1-3-2). Twenty-four hours later they briefly rehearsed the sequence (reactivating it) and learned a second sequence (e.g., 2-31-4). When tested on Day 3, accuracy performance for Sequence 1 was significantly impaired in comparison to a group of participants who did not rehearse Sequence 1 before learning Sequence 2 . This shows that the reactivation of the memory for Sequence 1 on Day 2 destabilized it such that a competing motor pattern could interfere.

Galluccio (2005) and Galluccio and Rovee-Collier (2005) investigated the fate of reactivated memories in infants trained to kick their foot to activate a mobile. After a delay, infants were reminded of the event: The moving mobile was presented for a brief period during which it was no longer attached to the baby's foot. After reactivation, one group of infants learned to move a novel mobile. One day later, infants who were exposed to the novel mobile showed a modification of the reactivated memory in that they no longer recognized the original mobile and solely reacted to the novel one.

Thus, reconsolidation effects have been shown in humans, but only for procedural memory (Walker et al. 2003) and conditioning (Galluccio 2005). These are both forms of implicit memory that do not require conscious recollection, making it important to ask whether reconsolidation also applies to explicit memory, a form of memory that allows for the conscious recollection of events (episodic memory) and facts (semantic memory).

Although the idea of fixed consolidated memory traces has been the dominant view in neurobiology, it has long been known in cognitive psychology that episodic memories can be reconstructed. For instance, Bartlett's (1932) classic study in which participants were repeatedly asked to recall a Native American folktale showed that, over time, participants reconstructed their recall to better fit their own cultural schema. It is also well known, from the study of retroactive interference, that a subsequent learning experience can impair performance for previously acquired material (e.g., Barnes and Underwood 1959; Delprato 2005). And we know that episodic memories can be 
Table 1. Lists of objects presented on Day 1 and Day 2

\begin{tabular}{ll}
\hline List $\mathbf{1}$ & \multicolumn{1}{c}{ List $\mathbf{2}$} \\
\hline Balloon & Apple \\
Bow & Band-aid \\
Calculator & Battery \\
Toy car & Book \\
Crayon & Cassette tape \\
Cup & Cellular phone \\
Dice & Comb \\
Feather & Dollar bill \\
Flashlight & Elephant \\
Flower & Envelope \\
Glue & Paper clip \\
Key & Toy pot \\
Sock & Puzzle piece \\
Sponge & Rock \\
Spoon & Straw \\
Sunglasses & Thread \\
Teabag & Tissue \\
Tennis ball & Watch \\
Toothbrush & Shovel \\
Whistle & Zipper \\
\hline
\end{tabular}

altered by post-event information. For instance, providing participants with misleading information after the encoding of an event can revise the original memory (misinformation effect; e.g., Loftus 2005). In contrast to those previous studies, the design and hypotheses of our study were directly derived from recent studies in neurobiology. Based on the reconsolidation findings we hypothesized that the modification of consolidated episodic memories critically depends upon their reactivation. In this way, our study tries to bridge the gap between recent studies in neurobiology and a long-standing tradition in cognitive psychology. We will consider the similarities and differences between previous cognitive studies and our findings in more detail in the General Discussion.

In our study, college students were taught a list of items on Day 1. After a reminder (or no reminder) on Day 2, they were taught a second list of items. Memory performance for the original list was tested on Day 3. If reminding puts the memory of List 1 (Table 1) into a labile state, then immediate learning of List 2 (Table 1) should alter memory for List 1 . It is important to note that the reconsolidation account makes no predictions about the direction of influence. It simply states that reactivation reopens memories for possible modification. The direction of influence is determined by the post-reminder manipulation. Thus, memories could be strengthened, weakened, or new information could be incorporated. The latter effect would reflect a constructive rather than destructive effect of reconsolidation.

We reactivated memory in a more subtle way than in the Walker et al. (2003) and Galluccio (Galluccio 2005; Galluccio and Rovee-Collier 2005) studies. In the latter cases, participants were re-presented with the actual learned material. We asked participants to describe the learning procedure without asking them to explicitly recall the learned items in order to test whether an incidental reminder can reactivate memory.

\section{Materials and Methods}

\section{Experiment 1}

\section{Design and participants}

The only independent variable that was varied between subjects was the procedure on Day 2: Before learning the second list, subjects were either reminded or not reminded of the learning session that had taken place on Day 1 (reminder and noreminder group, respectively), and a third group of subjects omitted Day 2 altogether (interference control). This group was in- cluded as a comparison to the experimental groups to ascertain the possible interfering effect of List 2 on memory for List 1 regardless of a reminder.

A total of 36 undergraduate students from the University of Arizona participated in the experiment. They received course credit for participation. Twelve subjects were randomly assigned to each condition.

\section{Materials}

List 1 and List 2 materials each consisted of 20 unrelated objects (see Table 1).

\section{Procedure}

The three sessions took place on Monday, Wednesday, and Friday of the same week. Participants were informed that they would have to memorize different lists of objects on the different days. Students participated one at a time.

On Day 1, the experimenter pulled out one item at a time from a bag and placed it in a distinctive blue basket (objects were from List 1). Participants were asked to name each item and to pay close attention so they could remember the items later. After all 20 items were placed into the basket the experimenter hid the basket and asked the participants to remember as many items as possible. This procedure was repeated until the participants remembered at least 17 of the 20 objects or until a maximum of four learning trials was reached.

The procedure on Day 2 differed for the three experimental groups. For participants in the reminder group, the same experimenter who administered the procedure on Day 1 showed them the empty blue basket and asked, "Do you remember this basket and what we did with it?" Participants were encouraged to describe the procedure, but were stopped if they started to recall any specific items. For participants in the no-reminder group a new experimenter administered the experimental procedure in a different room. The experimenter did not ask what had happened on Day 1 nor did she present the basket. The interference control participants omitted Day 2.

Participants in both the reminder and the no-reminder groups were asked to learn a second list of 20 objects (List 2). The procedure differed from that of Day 1 so the task would not serve as a reminder. All objects were placed in front of the participants, who were asked to name each of the objects, and were given 30 sec to study them. The experimenter then removed the objects and asked the participants to recall as many of the objects as possible. If participants recalled $<17$ objects, the objects were placed in front of the participant for another $30 \mathrm{sec}$. This was repeated until participants recalled at least 17 objects, or for a maximum of four learning trials.

On Day 3, the experimenter from Day 1 asked the participants to recall as many objects as possible from Day 1 , and the experimenter noted the remembered objects. When participants indicated that they could not remember any more items, the experimenter engaged the participants in a conversation about an unrelated topic for about $30 \mathrm{sec}$. The experimenter repeated the recall test by asking the participants to recall the objects again. This procedure was repeated for a total of four consecutive recall trials in order to test reliability of recall. The recall session took $\sim 15$ min to complete.

\section{Results}

\section{Performance on Day 1: Acquisition of List 1}

We recorded how many learning trials were necessary for participants to recall at least 17 objects. Participants who recalled $<17$ objects during the fourth learning trial were given a score of 5 . Participants took on average $3.11(S D=1.26)$ learning trials to 
reach criterion. There were no differences between the three groups $\left(F_{(2,33)}=2.40, M S E=1.47, P=0.11\right)$.

\section{Performance on Day 2: Acquisition of List 2}

Participants took on average $3.79(S D=1.38)$ learning trials to reach criterion. There was no difference between the reminder and the no-reminder group $(t<1)$.

\section{Performance on Day 3: Recall}

The mean percent of items recalled from List 1 (averaged over all four recall trials) and the mean percent of items falsely recalled from List 2 (intrusions, averaged over all four recall trials) are displayed in Figure 1. Although analyses were conducted on number of items, we report mean percentages in figures to facilitate cross-experiment comparisons.

\section{List 1 recall}

The number of objects recalled from List 1 was analyzed with a $3 \times 4$ mixed Analysis of Variance (ANOVA) with group (reminder, no-reminder, interference control) as the betweensubjects variable and trial (1-4) as the within-subjects variable. Because the sphericity assumption was violated, we report Huynh-Feldt corrected values. The ANOVA revealed a main effect of trial $\left(F_{(2.48,81.75)}=11.12, M S E=1.78, P<0.01\right.$; all other effects, $F \leq 1.74, P \geq 0.19)$. Within-subjects contrasts revealed a linear trend such that recall performance increased across trials, replicating previous studies (cf. Howe and Brainerd 1989) $\left(F_{(1,33)}=18.01, M S E=2.47, P<0.01\right.$, all other trends, $F \leq 2.85$, $P \geq 0.10)$.

\section{Intrusions from List 2}

The number of intrusions from List 2 was analyzed with a 3 (group) $\times 4$ (trial) mixed ANOVA. We found only a main effect of group $\left(F_{(2,33)}=21.84, M S E=13.39, P<0.01\right.$; all other effects, $F \leq 1.43, P \geq 0.25$ ). Post hoc comparisons (Dunnett's $C$ ) revealed that participants in the reminder group showed significantly more intrusions of items from List 2 in their recall of List 1 than participants in the no-reminder group or participants in the interference control. There was no significant difference in the amount of intrusions between the no-reminder group and the interference control.

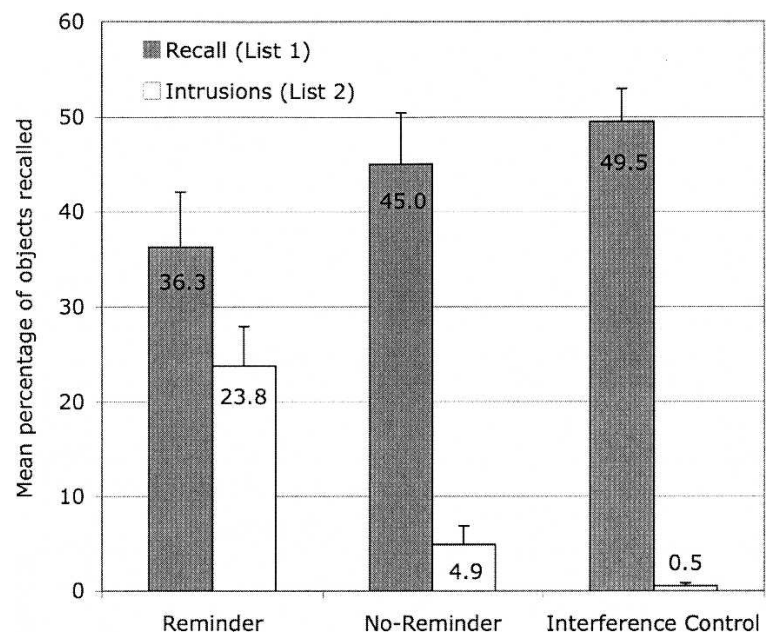

Figure 1. Mean percentage of objects correctly and falsely recalled on Day 3 (Experiment 1) in the reminder, the no-reminder, and the interference control group during Experiment 1 (recall test on Day 3). Error bars represent standard errors of means. Note: Participants were asked to recall objects from List 1. Objects that were falsely recalled from List 2 are labeled as Intrusions.
Because the no-reminder group showed few intrusions (floor effects), and the variance was higher in the reminder than in the no-reminder group, we ran an additional nonparametric test with the mean number of intrusions recalled by each participant over the four test trials serving as the dependent variable. A Mann-Whitney $U$-test detected significant differences between the reminder and the no-reminder group $(U=20.00, P<0.01)$.

\section{Discussion}

The experimental groups did not differ in the number of objects recalled from List 1 on Day 3, showing that the reminder did not strengthen or weaken memory of the original list, and that learning a second list had no lasting impact on memory for items on List $1 .{ }^{4}$ However, the reminder did reactivate memory of the original list, returning it to a state in which new information could be incorporated: When asked to recall List 1 on Day 3, participants in the reminder group incorrectly intermixed a high number of objects from List 2 in their recall of List 1 . In contrast, participants in the no-reminder group showed few intrusions.

Our results parallel Walker et al.'s (2003) findings on reconsolidation in human procedural memory and suggest that explicit memory processes are subject to the same updating process. Nader et al. (2005) propose a reconsolidation process involving three steps: (1) Reactivation of the existing memory returning it to a labile state, (2) modification of the existing memory, and (3) reconsolidation of the modified memory over a period of time. While Experiment 1 clearly demonstrates the first two steps (the reminder reactivated the memory for the original list, and the presentation of the new list modified the existing memory), an open question is whether memory was altered immediately or whether, as assumed by Nader (2003), memory modification involves a time-dependent reconsolidation process. If so, we should see no difference between the reminder and the noreminder group in a memory test administered immediately after presentation of the second list on Day 2 (as shown by Walker et al. 2003). We addressed this issue in Experiment 2.

\section{Experiment 2}

We tested whether a reminder has an immediate effect on memory or whether memory modification occurs over time. Participants were asked to recall List 1 directly after they had learned List 2 on Day 2. If the reminder-induced modification effects are instant, we should replicate the findings of Experiment 1, i.e., we should see more intrusions in the reminder than in the noreminder group. If, as suggested by the reconsolidation account, reactivated memories need to undergo a time-dependent reconsolidation process, we might not find any immediate differences between the reminder and no-reminder group.

\section{Materials and Methods}

Experiment 2 employed exactly the same materials and experimental groups, and followed exactly the same procedure as Experiment 1 with one exception: The experiment consisted of only two experimental sessions (Monday, Wednesday). The procedure described for Day 3 in Experiment 1 was administered on Day 2 immediately after the reminder and no-reminder group had learned List 2.

${ }^{4}$ One could raise the objection that our failure to find differences in List 1 recall stemmed from lack of statistical power. However, we recently replicated this effect in 5- and 9-year-old children. In these age groups, the numerical difference in the recall of List 1 items between the reminder and the no-reminder groups was much smaller. Given that all other effects were replicated in the younger age groups, we are confident that the null effect in Experiment 1 was not simply due to a lack of statistical power. 


\section{Participants}

A total of 36 undergraduate students from the University of Arizona who had not taken part in Experiment 1 participated in Experiment 2. They received course credit for participation. Twelve subjects were randomly assigned to each of the three conditions (reminder, no reminder, interference control).

\section{Results}

\section{Performance on Day 1: Acquisition of List 1}

Participants took on average $3.17(S D=1.08)$ learning trials to recall at least 17 objects. The three groups did not differ in the amount of learning trials $\left(F_{(2,33)}=1.54, M S E=1.14, P=0.23\right)$.

\section{Performance on Day 2: Acquisition of List 2}

Participants in the reminder group $(M=2.50, S D=0.91)$ learned List 2 with fewer trials than participants in the no-reminder group $\left(M=4.00, S D=1.21, t_{(22)}=3.45, P<0.01\right)$.

\section{Performance on Day 2: Recall}

The mean number of items recalled from List 1 and the mean number of items falsely recalled from List 2 (intrusions) are displayed in Figure 2.

\section{List 1 recall}

The number of objects recalled from List 1 was analyzed with a 3 (group) $\times 4$ (trial) mixed ANOVA as in Experiment 1 . The ANOVA revealed a significant main effect of group $\left(F_{(2,33)}=17.55, M S E=31.00, P<0.01\right)$. Post hoc comparisons (Dunnett's C) showed that the interference control group recalled significantly more items than the reminder and the noreminder groups. The latter two groups did not differ in their performance. Additionally, the main effect of trial was significant $\left(F_{(2.78,91.56)}=14.72, M S E=1.46, P<0.01\right.$ [Huynh-Feldt corrected values]). Within-subjects contrasts revealed a linear trend such that recall performance increased across trials $\left(F_{(1,33)}=26.23\right.$, MSE $=2.25, P<0.01$; all other trends, $F<1$.

\section{Intrusions from List 2}

The number of intrusions from List 2 was analyzed with a 3 (group) $\times 4$ (trial) mixed ANOVA. None of the effects were significant $(F \leq 2.37, P \geq 0.11)$.

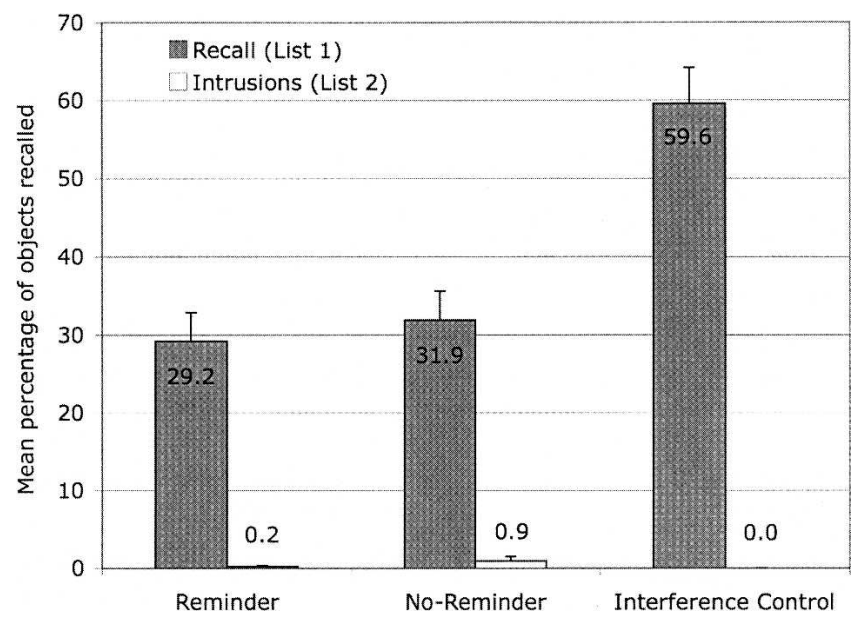

Figure 2. Mean percentage of objects correctly and falsely recalled on Day 2 (Experiment 2) in the reminder, the no-reminder, and the interference control group. Error bars represent standard errors of means. Note: Participants were asked to recall objects from List 1. Objects that were falsely recalled from List 2 are labeled as Intrusions.

\section{Discussion}

The reminder clearly had no immediate effect on memory for List 1: When asked to recall List 1 on Day 2, the reminder and the no-reminder groups did not differ in the number of objects recalled from List 1 and, more importantly, in both groups, the number of intrusions from List 2 was very low.

The interference control group that did not learn a second list at all recalled more objects from List 1 than the reminder and the no-reminder group. This effect could reflect a general retroactive interference effect. However, because this effect only occurred when memory for List 1 was tested immediately after learning List 2 (Experiment 2), and not on a later memory test (Experiment 1), the effect might be due to a nonspecific effect of fatigue resulting from learning a second list right before recall of the first list. A third explanation is that the difference reflects an extra load on participants who may have been actively suppressing the List 2 items they had just learned during their recall of List 1. The latter two explanations are consistent with the fact that the reminder and no-reminder groups recalled fewer items from List 1 when tested immediately (Experiment 2) than when tested after a delay (Experiment 1). Alternately, the better recall after a longer time delay (Experiment 1) could be the result of spontaneous recovery from retrieval inhibition that was caused by the preceding learning of List 2 (e.g., Wheeler 1995).

Importantly, the results of Experiment 2 suggest that the reminder effect found in the first experiment does not occur immediately (as would be predicted if this were retroactive interference). Rather, as predicted by a reconsolidation account, the effect evolves over time.

\section{Experiment 3}

One alternative explanation for the reminder effect found in Experiment 1 is that the reminder group had difficulties differentiating between List 1 and 2 because both lists were learned in the same context. Participants in the reminder group learned List 2 in the same spatial context as List 1. Additionally, the same experimenter who had instructed them on Day 1 administered the task on Day 2. In contrast, participants in the no-reminder group were presented with List 2 in a different room and were instructed by a different experimenter. According to a sourcemonitoring account (Johnson et al. 1993), the more features memories for different events have in common, the more likely source errors are to occur. Thus, the similarity in environmental context during encoding of List 1 and 2 in the reminder group could have led to source confusions. However, contextual distinctiveness does not always help specify the source of a memory. For example, studies in the misinformation paradigm show that the similarity of the environmental context in which event and post-event information is presented has no significant impact on the misinformation effect (e.g., Bonto and Payne 1991; Mitchell and Zaragoza 2001), thus similarities in the environmental context of List 1 and List 2 learning may not necessarily explain our findings.

Nevertheless, if the reminder effect simply reflects sourcemonitoring difficulties, participants should just as likely intermix List 1 items in their recall of List 2 as they intermixed List 2 items into their recall of List 1 in Experiment 1 . This was tested in Experiment 3. Participants were asked to recall List 2 on Day 3 instead of List 1.

\section{Materials and Methods}

Experiment 3 employed exactly the same materials and followed exactly the same procedure as Experiment 1 with two exceptions: (1) Participants were asked to recall List 2 instead of List 1 on Day 3 , and (2) we did not include a no-interference control group. 


\section{Participants}

A total of 24 undergraduate students from the University of Arizona who had not taken part in either of Experiments 1 or 2 participated in Experiment 3. They received course credit or financial compensation for their participation. Twelve subjects were randomly assigned to each condition (reminder, noreminder).

\section{Results}

\section{Performance on Day 1: Acquisition of List 1}

Participants took on average $3.75(S D=1.03)$ learning trials to recall at least 17 objects. The groups did not differ in the amount of learning trials $(t<1)$.

\section{Performance on Day 2: Acquisition of List 2}

Participants took on average $3.33(S D=1.40)$ learning trials to recall at least 17 objects. The groups did not differ in the amount of learning trials $(t<1)$.

\section{Performance on Day 3: Recall}

The mean number of items recalled from List 2 and the mean number of items falsely recalled from List 1 (intrusions) are displayed in Figure 3.

\section{List 2 recall}

The number of objects recalled from List 2 was analyzed with a 2 (group) $\times 4$ (trial) mixed ANOVA. The ANOVA revealed only a significant main effect of trial $\left(F_{(2.09,45.93)}=8.37, M S E=1.75\right.$, $P<0.01$ [Huynh-Feldt corrected values]; no other effects were significant $[F<1])$. Within-subjects contrasts revealed a linear trend such that recall performance increased across trials $\left(F_{(1,22)}=13.48, M S E=2.26, P<0.01\right.$; all other trends, $\left.F<1\right)$.

\section{Intrusions from List 1}

The number of intrusions from List 2 was analyzed with a 2 (group) $\times 4$ (trial) mixed ANOVA. None of the effects were significant $(F \leq 2.13, P \geq 0.16)$. Additionally, we compared the number of intrusions for the reminder groups of Experiment 1 and 3 with a 2 (Experiment 1 vs. Experiment 3$) \times 4$ (trial) mixed ANOVA. We found a significant main effect of experiment $\left(F_{(1,22)}=14.57, M S E=22.14, P<0.01\right)$, showing that the reminder group in Experiment 1 (recall of List 1) showed signifi-

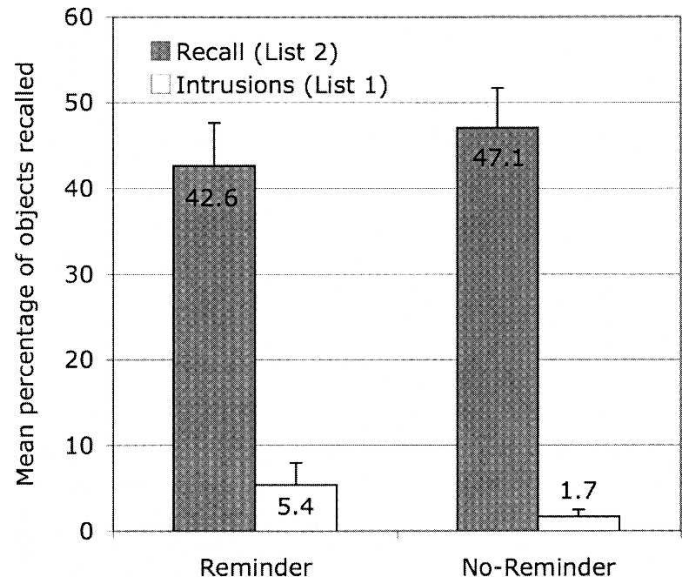

Figure 3. Mean percentage of objects correctly and falsely recalled from List 2 on Day 3 (Experiment 3) in the reminder and the no-reminder groups. Error bars represent standard errors of means. Note: Participants were asked to recall objects from List 2 . Objects that were falsely recalled from List 1 are labeled as Intrusions. cantly more intrusions than the reminder group in Experiment 3 (recall of List 2).

\section{Discussion}

Experiment 3 shows that the reminder had no effect on recall of List 2. Participants in the reminder group intermixed items from List 2 into List 1 (Experiment 1), but they showed few intrusions from List 1 into List 2 recall (Experiment 3). The 5.4\% intrusion rate in Figure 3 represents an average of one item out of a possible 20. If participants had been generally uncertain about the source (i.e., the list) of the items they recalled, List 1 and 2 should have been equally affected. Although participants in the reminder group of Experiment 1 clearly misattributed the source of many List 2 items as being from List 1, this source error is a rather unusual one because it is asymmetric and because it contradicts the assumption that, with time delay, source errors should increase. Because List 1 was learned two days before List 2, participants should have been more likely to confuse the source of List 1 than List 2 items.

Walker et al. (2003) reported similar findings for the procedural motor-skill task: As in Experiment 3, memory for the second sequence was not affected by a brief rehearsal of the first sequence. However, like Experiment 1, the second sequence negatively affected memory of the first sequence when the first was rehearsed prior to learning the second one.

Taken together, Experiments 1 and 3 show that the reminder uniquely affected the reactivated memory for List 1 thus eliminating simple source-monitoring difficulties as an explanation.

\section{General Discussion}

The present study demonstrates reconsolidation effects in human episodic memory. Although many have suggested that memory storage is dynamic (e.g., Tulving 1984; Loftus 2005), until recently reactivation as a prerequisite for memory change was not considered. We show that the modification of episodic memories depends critically upon their preceding reactivation as suggested by the reconsolidation account.

Similar to what has been found for Pavlovian conditioning (e.g., Nader et al. 2000), instrumental conditioning (e.g., Wang et al. 2005), and human procedural memory (Walker et al. 2003), reactivated episodic memories also undergo a time-dependent reconsolidation process: Incorporation of new information did not occur immediately but was seen two days after memory reactivation and subsequent presentation of new material.

The finding, that stored memories remain open to change as a function of related experience, suggests that the paradigmatic assumption in memory research of a "fixed" trace or "engram" is in need of modification. We have recently proposed a different kind of memory model-the "multiple trace theory" (MTT) (Nadel and Moscovitch 1997; Moscovitch and Nadel 1998; Moscovitch et al. 2005), which takes as given that reactivating a previously "stored" memory leads to the creation of a new version of that memory. MTT was originally proposed to account for anomalous results in the study of memory consolidation (e.g., its long duration), prior to the re-emergence of the reconsolidation account. However, the characteristics of reconsolidation mesh well with MTT and provide strong support for this alternative account to the standard version of memory consolidation.

Although reconsolidation is a rather new concept in neurobiology, the idea that episodic memory traces can be modified by post-event information has long been accepted in cognitive psychology. The most widely studied phenomena illustrating the reconstructive nature of episodic memory are retroactive inter- 
ference and misinformation. What are the similarities and differences between those effects and the present reminder effect? The most obvious similarity is in regard to the effects themselves where recall of the originally encoded information is affected by the interpolated presentation of new information. However, there are also important differences in the paradigms and effects, which we will now consider.

The classic retroactive interference paradigm involves learning a list of stimulus pairs (A-B) followed by subsequent learning of stimulus pairs of the form A-D, i.e., the old stimulus A is paired with a new stimulus $D$ in the second list. Later, participants are asked to recall either B or D or both in response to the cue word A. The classic finding is that, with an increasing number of interpolated learning trials $(\mathrm{A}-\mathrm{D})$, recall of $\mathrm{B}$ diminishes and recall of D increases (e.g., Barnes and Underwood 1959). It is assumed that both unlearning of A-B associations and response competition between B and D underlie this effect (e.g., Postman and Underwood 1973).

Although the interference paradigm resembles our paradigm at first sight, there are two major differences. First, learning of A-D usually takes place right after learning of A-B, i.e., the interference paradigm lacks the crucial reactivation component. Therefore, even if one argues that A serves as a reminder of $\mathrm{B}$, the classic retroactive interference paradigm speaks to the issue of memory consolidation (cf., Müller and Pilzecker 1900) and not to reconsolidation. However, in studies with a time delay between the presentation of A-B and learning of A-D, A could well serve as a reminder of $B$ and, in turn, reconsolidation processes could play a role in the unlearning of $\mathrm{B}$. Because interference as well as misinformation studies do not usually include a noreactivation condition, it is impossible at this point to decide what role reconsolidation processes might play in the development of such effects. Second, we did not replicate classic interference effects. A comparison between the no-reminder and the no-interference control in Experiment 1 shows that learning a second list does not affect memory for the first list per se. Our critical effect was dependent on the presence of a reminder. While the reminder caused participants to incorporate List 2 items into List 1 recall, it did not significantly diminish recall of List 1 items.

The misinformation paradigm is very similar to the retroactive interference paradigm. In misinformation studies (e.g., Loftus 1975; for a recent review, see Loftus 2005), participants are presented with a complex event (e.g., a slide show depicting a car accident). Then, half of the participants receive some misleading information about the event (e.g., about the traffic sign that marked the intersection), whereas the other half does not. When participants are later asked to remember the event, the misled participants often report the misinformation (e.g., a yield sign) instead of the information that was part of the slide show (e.g., a stop sign). Loftus (1975) assumed that the misleading information alters the original memory trace (cf. unlearning in retroactive interference). Alternatively, McCloskey and Zaragoza (1985) proposed that the original memory trace remains unaffected. Rather, misinformation can be accepted for a variety of reasons. For instance, participants might not have encoded the original information sufficiently or might have difficulties remembering it, and the misinformation simply fills in those gaps. Participants might also confuse the source of misinformation as being from the original event (cf. source-monitoring account; Johnson et al. 1993).

We hypothesize that reconsolidation is the mechanism that mediates the misinformation effect. In the misinformation paradigm, the misleading information is directly related to the original event. For instance, the misleading information is often embedded in questions about the original event (e.g., how fast was the car that ran the yield sign). Thus, it is likely that the misleading information reactivates the original memory, making it open to change. However, a condition in which the memory is not reactivated is usually missing in the misinformation paradigm (for an exception and a reduced false memory effect, see Lindsay et al. 2004). Such a control condition would need to be incorporated in the misinformation paradigm to directly compare the misinformation effect and reconsolidation.

Reconsolidation is an important mechanism for understanding plasticity, potentially explaining how organisms build on prior experience while incorporating new information. Although previous studies have mainly demonstrated disruptive effects of reactivation, our study shows that reminders can also trigger constructive processes, allowing for the incorporation of new information into old memories. Our study also shows that memory can be activated with a fairly subtle reminder. An interesting question has to do with the cues triggering reactivation: recall of the first session or reminding from experimental context (the location of testing and/or the experimenter). We are currently exploring this issue.

In conclusion, we have demonstrated post-reminder effects in episodic memory. More than a hundred years ago, Müller and Pilzecker (1900) suggested a perseveration-consolidation process to explain their observations that retroactive interference decreased with the amount of elapsed time between the presentation of the first and second list. We now show that a second list can have memory-changing effects even at a later time point and that this effect depends critically upon the reactivation of memory for the original list. This finding suggests that memory "traces" are dynamic rather than static, and that consolidation does not irreversibly transform them into a permanent state.

\section{Acknowledgments}

We thank Auna Lee Otts for helping with data collection. This research was funded by NSF CAREER award BCS-0238584 to the second author. The first author is partially supported by the Arizona Alzheimer's Research Consortium.

\section{References}

Alberini, C.M. 2005. Mechanisms of memory stabilization: Are consolidation and reconsolidation similar or distinct processes? Trends Neurosci. 28: 51-56.

Barnes, J.M. and Underwood, B.J. 1959. "Fate" of first-list-associations in transfer theory. J. Exp. Psychol. 58: 97-105.

Bartlett, F.C. 1932. Remembering: A study in experimental and social psychology. Cambridge University Press, Cambridge.

Bonto, M.A. and Payne, D.G. 1991. Role of environmental context in eyewitness memory. Am. J. Psychol. 104: 117-134.

Delprato, D.J. 2005. Retroactive interference as a function of degree of interpolated study without overt retrieval practice. Psychon. Bull. Rev. 12: $345-349$.

Dudai, Y. and Eisenberg, M. 2004. Rites of passage of the engram: Reconsolidation and the lingering consolidation hypothesis. Neuron 44: $93-100$.

Galluccio, L. 2005. Updating reactivated memories in infancy: I. Passiveand active-exposure effects. Dev. Psychobiol. 47: 1-17.

Galluccio, L. and Rovee-Collier, C. 2005. Updating reactivated memories in infancy: II. Time passage and repetition effects. Dev. Psychobiol. 47: 18-30.

Howe, M.L. and Brainerd, C.J. 1989. Development of children's long-term retention. Dev. Rev. 9: 301-340.

Johnson, M.K., Hashtroudi, S., and Lindsay, D.S. 1993. Source monitoring. Psychol. Bull. 114: 3-28.

Lewis, D.J., Misanin, J.R., and Miller, R.R. 1968. The recovery of memory following amnestic treatment. Nature 220: 704-705.

Lindsay, D.S., Allen, B.P., Chan, J.C.K., and Dahl, L.C. 2004. Eyewitness suggestibility and source similarity: Intrusions of details from one event into memory reports of another event. J. Mem. Lang. 50: $96-111$.

Loftus, E.F. 1975. Leading questions and the eyewitness report. Cognit. Psychol. 7: 560-572.

Loftus, E.F. 2005. Planting misinformation in the human mind: A

\section{Learning \& Memory


30-year investigation of the malleability of memory. Learn. Mem. 12: $361-366$.

McCloskey, M. and Zaragoza, M. 1985. Misleading postevent information and memory for events: Arguments and evidence against memory impairment hypotheses. J. Exp. Psychol. Gen. 114: $1-16$.

McGaugh, J.L. 2000. Memory-A century of consolidation. Science 287: 248-251.

Misanin, J.R., Miller, R.R., and Lewis, D.J. 1968. Retrograde amnesia produced by electroconvulsive shock after reactivation of a consolidated memory trace. Science 160: 554-555.

Mitchell, K.J. and Zaragoza, M.S. 2001. Contextual overlap and eyewitness suggestibility. Mem. Cognit. 29: 616-626.

Moscovitch, M. and Nadel, L. 1998. Consolidation and the hippocampal complex revisited: In defense of the multiple-trace model. Curr. Opin. Neurobiol. 8: 297-300.

Moscovitch, M., Rosenbaum, R.S., Gilboa, A., Addis, D.R., Westmacott, R., Grady, C., McAndrews, M.P., Levine, B., Black, S., Winocur, G., et al. 2005. Functional neuroanatomy of remote episodic, semantic and spatial memory: A unified account based on multiple trace theory. $J$. Anat. 207: 35-66.

Müller, G.E. and Pilzecker, A. 1900. Experimentelle Beiträge zur Lehre vom Gedächtnis. Z. Psychol. 1: 1-300.

Nadel, L. and Moscovitch, M. 1997. Memory consolidation, retrograde amnesia and the hippocampal complex. Curr. Opin. Neurobiol. 7: 217-227.

Nader, K. 2003. Memory traces unbound. Trends Neurosci. 26: 65-72.
Nader, K., Schafe, G.E., and Le Doux, J.E. 2000. Fear memories require protein synthesis in the amygdala for reconsolidation after retrieval. Nature 406: 722-726.

Nader, K., Hardt, O., and Wang, S.-H. 2005. Response to Alberini: Right answer, wrong question. Trends Neurosci. 28: 346-347.

Postman, L. and Underwood, B.J. 1973. Critical issues in interference theory. Mem. Cognit. 1: 19-40.

Przybyslawski, J. and Sara, S.J. 1997. Reconsolidation after reactivation of memory. Behav. Brain Res. 84: 241-246.

Sara, S.J. 2000. Retrieval and reconsolidation: Toward a neurobiology of remembering. Learn. Mem. 7: 73-84.

Tulving, E. 1984. Precis of elements of episodic memory. Behav. Brain Sci. 7: 223-268.

Walker, M.P., Brakefield, T., Hobson, J.A., and Stickgold, R. 2003. Dissociable stages of human memory consolidation and reconsolidation. Nature 425: 616-620.

Wang, S.-H., Ostlund, S.B., Nader, K., and Balleine, B.W. 2005. Consolidation and reconsolidation of incentive learning in the amygdala. J. Neurosci. 25: 830-835.

Wheeler, M.A. 1995. Improvement in recall over time without repeated testing: Spontaneous recovery revisited. J. Exp. Psychol. Learn. Mem. Cogn. 21: 173-184.

Received July 11, 2006; accepted in revised form October 31, 2006. 


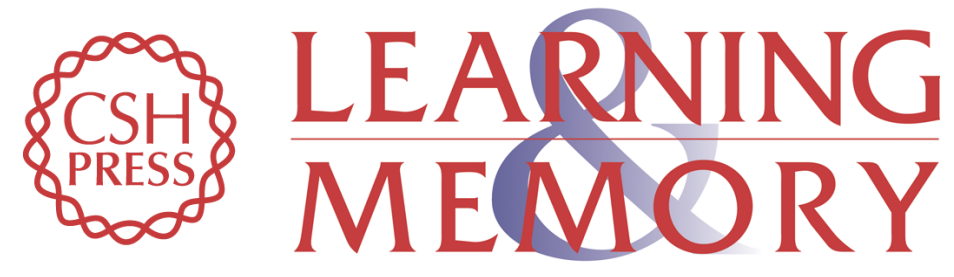

\section{Reconsolidation of episodic memories: A subtle reminder triggers integration of new information}

Almut Hupbach, Rebecca Gomez, Oliver Hardt, et al.

Learn. Mem. 2007, 14: originally published online January 3, 2007

Access the most recent version at doi:10.1101//m.365707

References

This article cites 31 articles, 5 of which can be accessed free at:

http://learnmem.cshlp.org/content/14/1-2/47.full.html\#ref-list-1

License

Email Alerting

Service
Receive free email alerts when new articles cite this article - sign up in the box at the top right corner of the article or click here. 